\title{
Stabilization System of a Bipedal non-anthropomorphic Robot AnyWalker
}

\author{
I. Ryadchikov ${ }^{1}$, S. Sechenev ${ }^{1}$, M. Drobotenko ${ }^{1}$, A. Svidlov ${ }^{1}$, P. Volkodav ${ }^{1}$, R. Vishnykov ${ }^{1}$, D. Sokolov ${ }^{2}$ \\ and E. Nikulchev ${ }^{3, *}$ \\ ${ }^{1}$ Kuban State University, Krasnodar, Russia \\ ${ }^{2}$ University of Lorraine, Nancy, France \\ ${ }^{3}$ MIREA - Russian Technological University, Moscow, Russia
}

Received 20 November 2017; Accepted 22 November 2018

\begin{abstract}
We present a bipedal walking non-anthropomorphic robot AnyWalker developed in the laboratory of robotics and mechatronics of the Kuban State University. The goal is to be able to overcome obstacles exceeding the size of the robot itself. In addition to the degrees of freedom due to the joints between the links, the robot is equipped with reaction wheels enhancing its dynamic stabilization capabilities. This paper presents a study of the stability zones in the frontal plane of the robot with and without the reaction wheel support.
\end{abstract}

Keywords: Robot, Stabilization System, Bipedal

\section{Introduction}

Currently, most of mobile robots are equipped with wheels or tracks for the locomotion. However, wheels and tracks are limited to good terrains only [1]. Movement through severely rugged terrain, climbing ladders and other obstacles motivate research in legged robots [2]. Walking robots are able to overcome complicated surfaces with large elevation angles without loss of stability, even if control poses a major problem [3]. Walking robots were already used to explore remote locations and hostile environments, such as the seabed, space, nuclear power plants and rescue operations [4].

In addition, legged locomotion allows for collecting materials, transporting goods, for moving to hard-to-reach areas of production and trunk pipelines, thus effectively allowing to create service robots. Let us cite the US Robotics 2016 Road Map report: «To extend the automation of the logistics chain into the world, robots must have mobility that matches human mobility - robots must negotiate stairs, elevators, doorways, curbs, broken concrete, cluttered environments, and go where people go. This type of advanced mobility is becoming realistic for robotic systems, legged and otherwise - and with such a solution, logistics will become fast, 24/7, on-demand, inexpensive, predictable, and well-tracked» [5].

The movement of walking systems is based on the principles of human and animal movement: step, running, jumping [7]. Threre are folloing disadvantages of walking robots: they include high complexity of control and stabilization, cost, low energy efficiency and relatively low speed [6]. We are mainly interested in bipedal walking robots, whose advantages is reduction of energy costs due to the use of natural oscillations. However, the stabilization problem is poorly solved due to the object's geometry complexity[8].

Another popular class of walking robots is six-legged mobile platforms with different types of chassis [4]. There is a large variety of studies that go from repeating kangaroo jumps [9] to tripartite systems [10].

In our laboratory, we develop the bipedal robot AnyWalker (Fig. 1). The robot has a spherical body moving with two triple link legs. Three links in each leg allow for a compact design without limiting zones the robot can reach. Bipedal design requires less servomotors to operate than designs with six legs.

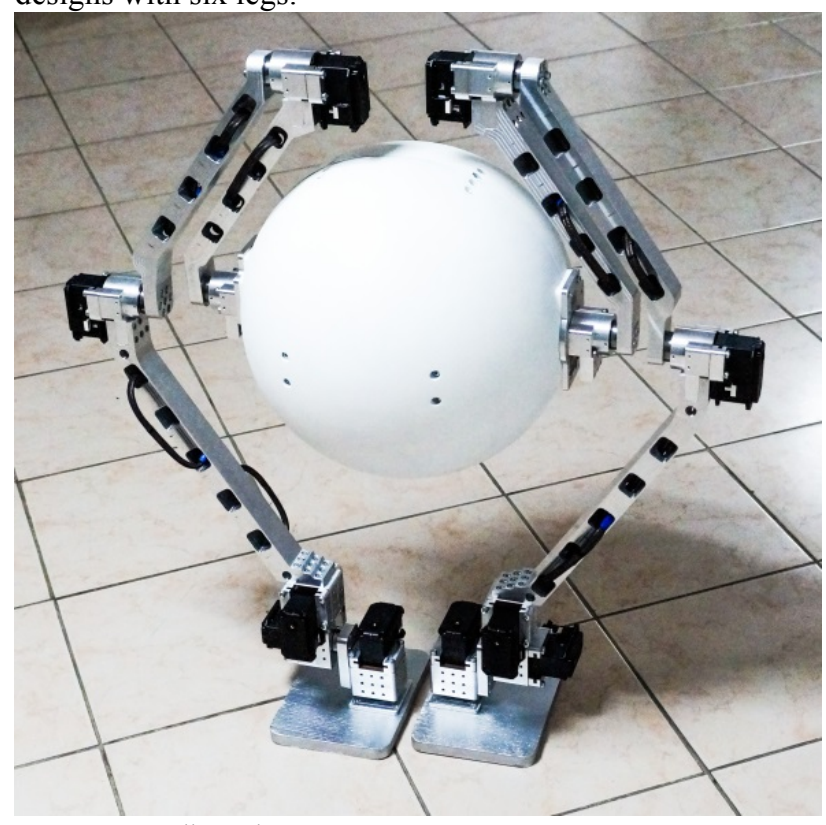

Fig. 1. AnyWalker robot

The distinctive feature of AnyWalker is the auxiliary stabilization system embedded in the spherical body of the robot [11]. Namely, the robot is equipped with three reaction wheels orthogonal one another. This system enhances 
dynamic stabilization capabilities of the robot. Studies of such systems have been widely carried out, for example in [12]. However, to the best of our knowledge, AnyWalker is the first bipedal robot equipped with an auxiliary stabilization system. Another key difference of the stabilization system is that the centers of inertia of the reaction wheels are combined at one point, leading to a better energy efficiency.

For the sake of simplicity, in this article, we only consider the stability in the frontal plane (one reaction wheel only). The approach presented in this paper can be extended to other planes.

\section{Robot Control System}

We are looking to expand the stability zone by adding a reaction wheel inside the robot's body. This section presents a study for the case where the robot is not allowed to change the position of its links relative to each other, i.e. it presents a form of an inverted pendulum. In this case the stability zone is defined by the maximum inclination angle that does not lead to an uncontrollable fall.

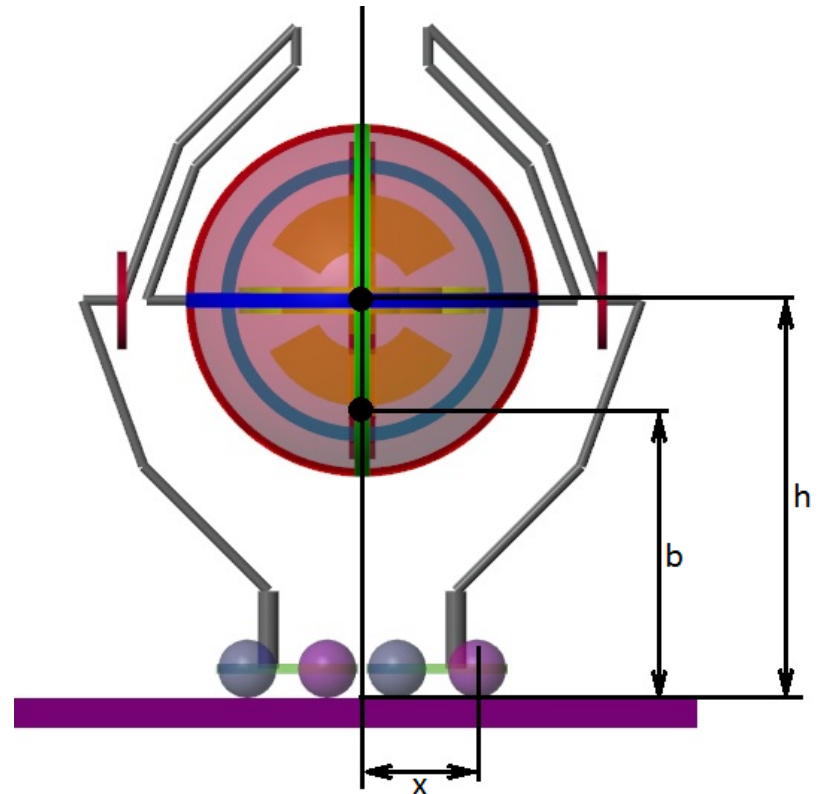

Fig. 2. The diagram of the chassis

Let us introduce following notations (Fig. 2):

$h$ - distance from the reaction wheel center to the ground or to the plane of support $[\mathrm{m}]$;

$x$-distance from the plane of symmetry to the outer edge of the robot support polygon [m];

$b$ - distance from the center of mass to the plane of support [m];

$J_{m}$ - moment of inertia of the robot's body (without the reaction wheel) around the center of the support polygon $[\mathrm{kg}$ $\cdot \mathrm{m} 2]$;

$J_{M}-$ moment of inertia of the reaction wheel w.r.t its axis of rotation $[\mathrm{kg} \cdot \mathrm{m} 2]$;

$m$ - mass of the robot without the reaction wheel $[\mathrm{kg}]$;

$M$ - reaction wheel mass $[\mathrm{kg}]$;

$g-$ free fall acceleration $\left[\mathrm{m} \cdot \mathrm{s}^{-2}\right]$;

$\left.\mathrm{A}^{-1}\right]$

$c_{u}$ - electric constant of the reaction wheel drive $[\mathrm{N} \cdot \mathrm{m} \cdot$

$c_{v}-$ mechanical drive constant of the reaction wheel $[\mathrm{N}$. $\mathrm{m} \cdot \mathrm{s}]$

$\omega(t)$ - reaction wheel angular speed $[\mathrm{rad} / \mathrm{s}] ;$

$u(t)$ - electric current supplied to the reaction wheel drive $[\mathrm{A}]$.

We study the robot's stability under a tilting action directed to the right, so let us define theangle $\beta(t)$ :

$\beta(t)$ - the angle between the AnyWalker axis and the "up" direction counter-clockwise (Fig. 3) ;

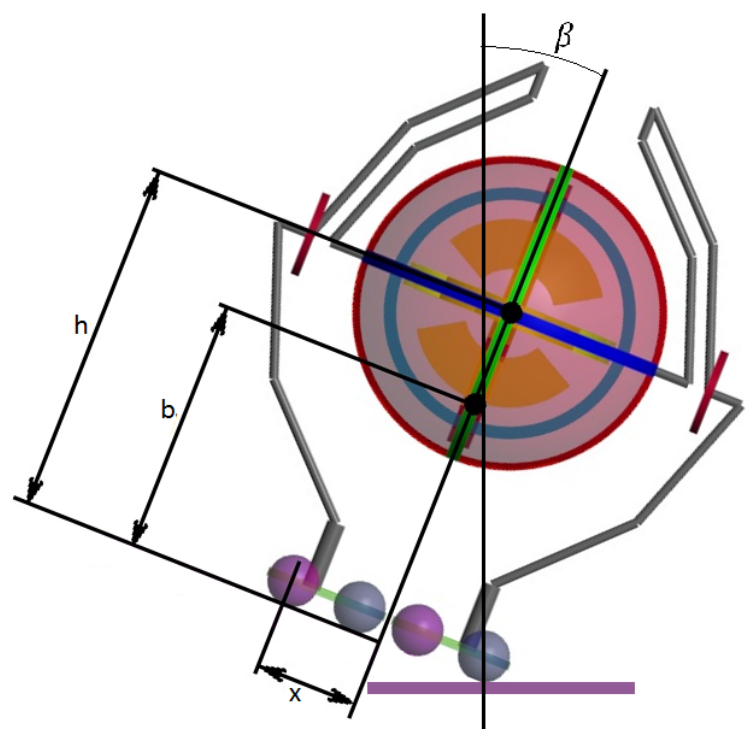

Fig. 3. Inclined position of the robot

To simplify the equations of motion, let us define constants $\mathrm{J}$ and $\mathrm{V}$ as:

$$
\begin{gathered}
x \\
\left(\mid 2+h^{2}\right)+J_{M}+m x^{2} \\
J=J_{m}+M \\
\mathrm{~V}=(m+M) \sqrt{x^{2}+b^{2}} g .
\end{gathered}
$$

The equations of motion are derived using the Lagrangian approach:

$J \dot{\beta}+J_{M} \dot{\omega}=V \sin \left(\beta-\operatorname{arctg} \frac{x}{b}\right)$,

$J_{M} \dot{\beta}+J_{M} \dot{\omega}=c_{u} u-c_{v} \omega$.

We linearize the equations around the equilibrium point $\beta=\operatorname{arctg} \frac{x}{b}$ :

$J \dot{\beta}+J_{M} \dot{\omega}=V\left(\beta-\operatorname{arctg} \frac{x}{b}\right)$,

$J_{M} \dot{\beta}+J_{M} \dot{\omega}=c_{u} u-c_{v} \omega$,

Then we can solve for $\dot{\beta}$ and $\dot{\omega}$ :

$\dot{\beta}=\frac{v\left(\beta-\operatorname{arctg} \frac{x}{b}\right)}{J-J_{M}}-\frac{c_{u} u-c_{v} \omega}{J-J_{M}}$,

$\dot{\omega}=\frac{c_{u} u-c_{v} \omega}{J_{M}-\frac{J_{M}^{2}}{J}}-\frac{v\left(\beta-\operatorname{arctg} \frac{x}{b}\right)}{J-J_{M}}$.

The state of our system is given by $\beta, \dot{\beta}$ and $\dot{\omega}$. In matrix form of the motion equations can be expressed as follows: 


$$
\begin{gathered}
\frac{d}{d t}\left(\begin{array}{c}
\beta \\
\dot{\beta} \\
\omega
\end{array}\right)=\left(\begin{array}{ccc}
0 & 1 & 0 \\
\frac{V}{J-J_{M}} & 0 & \frac{c_{v}}{J-J_{M}} \\
\frac{-V}{J-J_{M}} & 0 & \frac{-c_{v}}{J_{M}-\frac{J_{M}^{2}}{J}}
\end{array}\right)\left(\begin{array}{c}
\beta \\
\dot{\beta} \\
\omega
\end{array}\right)+\left(\begin{array}{c}
0 \\
\frac{-c_{u}}{J-J_{M}} \\
\frac{c_{u}}{J_{M}-\frac{J_{M}^{2}}{J}}
\end{array}\right) u \\
+\left(\begin{array}{c}
0 \\
-\frac{V}{J-J_{M}} \\
\frac{V}{J-J_{M}}
\end{array}\right) \operatorname{arctg} \frac{x}{b}
\end{gathered}
$$
A:

The behavior of the system is conditioned by the matrix

$$
A=\left(\begin{array}{ccc}
0 & 1 & 0 \\
\frac{V}{J-J_{M}} & 0 & \frac{c_{v}}{J-J_{M}} \\
\frac{-V}{J-J_{M}} & 0 & \frac{-c_{v}}{J_{M}-\frac{J_{M}^{2}}{J}}
\end{array}\right)
$$

Let us perform its spectral analysis. The characteristic polynomial of A has the following form:

$$
\begin{gathered}
F(\lambda)=\left|\begin{array}{ccc}
-\lambda & 1 & 0 \\
\frac{V}{J-J_{M}} & -\lambda & \frac{c_{v}}{J-J_{M}} \\
\frac{-V}{J-J_{M}} & 0 & \frac{-c_{v}}{J_{M}-\frac{J_{M}^{2}}{J}}-\lambda
\end{array}\right| \\
\left.=\left(\frac{-c_{v}}{J_{M}-\frac{J_{M}^{2}}{J}}\right) \lambda\right)\left(\lambda^{2}-\frac{V}{J-J_{M}}\right) \\
-\frac{c_{v}}{J-J_{M}} \frac{V}{J-J_{M}} .
\end{gathered}
$$

It is easy to see that the polynomial has three real roots: one positive root and two negative. Indeed, the polynomial is a continuous function, $F(\lambda) \rightarrow+\infty$ as $\lambda \rightarrow-\infty$. Moreover, $F(0)>0$, and $F\left(-\sqrt{\frac{V}{J-J_{M}}}\right)=F\left(\sqrt{\frac{V}{J-J_{M}}}\right)<0$. Therefore, it crosses the zero line thrice. The presence of a positive eigenvalue indicates the instability of a stationary point in the absence of control.

To simplify the equations further, we perform following change of variables:

$$
\alpha=\beta-\operatorname{arctg} \frac{x}{b}
$$

Then we obtain:

$$
\frac{d}{d t}\left(\begin{array}{c}
\alpha \\
\alpha \\
\omega
\end{array}\right)=\left(\begin{array}{ccc}
0 & 1 & 0 \\
\frac{V}{J-J_{M}} & 0 & \frac{c_{v}}{J-J_{M}} \\
\frac{-V}{J-J_{M}} & 0 & \frac{-c_{v}}{J_{M}-\frac{J_{M}^{2}}{J}}
\end{array}\right)\left(\begin{array}{c}
\alpha \\
\alpha \\
\omega
\end{array}\right)+\left(\begin{array}{c}
0 \\
\frac{-c_{u}}{J-J_{M}} \\
\frac{c_{u}}{J_{M}-\frac{J_{M}^{2}}{J}}
\end{array}\right) u .
$$

Let $\mathrm{x}$ be the state vector $x=(\alpha, \alpha, \omega)^{T}$ and $b=$
$\left(0,-\frac{c_{u}}{J-J_{M}}, \frac{c_{u}}{J_{M}-\frac{J_{M}^{2}}{J}}\right)^{T}$. Then the system of equations can be written as:

$$
\dot{x}=A x+b u .
$$

It is easy to see that the eigenvector corresponding to the eigenvalue $\lambda$ can be written as $\left(c_{v}, c_{v} \lambda,\left(J-J_{M}\right) \lambda^{2}-V\right)^{T}$. Let us define the matrix $\mathrm{K}$ whose columns are given by three corresponding eigenvectors:

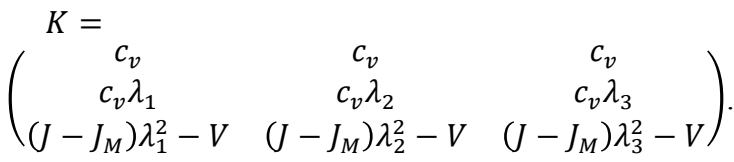

Without loss of generality, we assume that $\lambda_{1}>0>$ $\lambda_{2}>\lambda_{3}$. Under a second change of variables $x=K y$, we obtain:

$K \dot{y}=A K y+b u$

Then we multiply both parts of the equation by $K^{-1}$ :

$\dot{y}^{\prime}=K^{-1} A K y+K^{-1} b u=\operatorname{diag}\left(\lambda_{1}, \lambda_{2}, \lambda_{3}\right) y+K^{-1} b u$,

since $K^{-1} A K=\operatorname{diag}\left(\lambda_{1}, \lambda_{2}, \lambda_{3}\right)$.

We are interested by the stability zone under control constraint $u \mathrm{~V} \leq u_{\max }$.

If a system described by the equation

$\dot{y}=\operatorname{diag}\left(\lambda_{1}, \lambda_{2}, \lambda_{3}\right) y+K^{-1} b u$

starts from initial conditions with $y_{1} \geq\left|\left(K^{-1} b\right)_{1}\right| u_{\max } \lambda_{1}$, then $y_{1}$ will never decrease. Similarly, starting from a state with $y_{1} \leq-\left|m_{1}\right| u_{\max } \lambda_{1}, y_{1}$ will never increase.

There exists a control law $u=u(y, t)$ such that the solution of equation

$\dot{y}^{\prime}=\operatorname{diag}\left(\lambda_{1}, \lambda_{2}, \lambda_{3}\right) y+K^{-1} b u$,

from the state with $y_{1} \vee\left|m_{1}\right| u_{\max } / \lambda_{1}$ tends to a stationary point $(0,0,0)^{T}$.

Conclusion: the controllability domain is given by the inequality $y_{1} \vee\left|\left(K^{-1} b\right)_{1}\right| u_{\max } / \lambda_{1}$.

The maximum possible tilt angle is given by

$\beta_{\max }=\frac{\left|m_{1}\right| u_{\max }}{\left(K^{-1}\right)_{11} \lambda_{1}}+\operatorname{acrtg} \frac{x}{b}$

\section{Results of realization}

The reaction wheel of the Anywalker robot is driven by three Maxon EC-45 flat motors, so for the simulations on AnyWalker we have chosen following parameters according to the hardware:

$$
\begin{aligned}
& h-0,35[\mathrm{~m}] ; \\
& x-\text { from } 0 \text { to } 0.1[\mathrm{~m}] \\
& b-0.2[\mathrm{~m}] ; \\
& J_{m}-0.01[\mathrm{~kg} \cdot \mathrm{m} 2] ; \\
& J_{M}-0.005[\mathrm{~kg} \cdot \mathrm{m} 2] \\
& m-15[\mathrm{~kg}]
\end{aligned}
$$


$M-2[\mathrm{~kg}]$

$g-9.8\left[\mathrm{~m} \cdot \mathrm{s}^{-2}\right]$

$c_{u}-0.1107\left[\mathrm{~N} \cdot \mathrm{m} \cdot \mathrm{A}^{-1}\right]$

$c_{v}-0.00015[\mathrm{~N} \cdot \mathrm{m} \cdot \mathrm{a}]$

$u_{\max }-39.5 \mathrm{~A}$

Firstly, we study how to choose the size of the support polygon. To do so, we vary the width $\mathrm{x}$ and we plot maximum possible tilt angle (the tilt that does not lead to a fall). We plot two graphs: one with the reaction wheel active and the other one with the reaction wheel disabled. Fig. 4 shows the plots of the maximum angle vs. support polygon size. It can be seen that the smaller the width of the robot's feet, the higher the contribution of the reaction wheel to the stability of the robot.

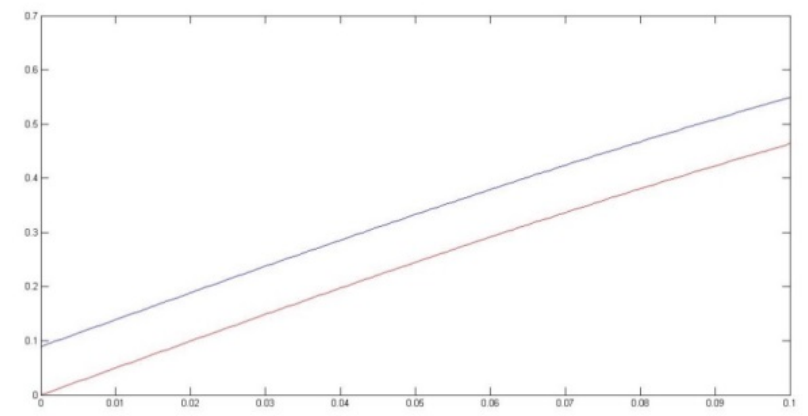

Thus, when the symmetry plane is $2 \mathrm{~cm}$ apart from the outer edge of the robot's feet, activation of the reaction wheel doubles the stability zone. If we choose $10 \mathrm{~cm}$ distance to the outer edge of the foot, activation of the reaction wheel increases the stability zone by $16 \%$.

Similarly, we can consider the stabilization problem of a robot in a posture ready to overcome the obstacles of maximum height. In this case, the equations of motion do not change, the only change is in the parameters $J_{m}$ and b (b $=\mathrm{h}$, and $\left.J_{m}=0.015[\mathrm{~kg} \cdot \mathrm{m} 2]\right)$. When the height of the center of mass above the support surface is increased. As a result, increase in the inertia moment leads to a shrink of the stability zone both for the cases with the reaction wheel active and disabled. Nevertheless, activation of the stabilization system expands the stabilization zone.

An example of a robot overcoming the step, with a height equal to the height of the robot is shown in Fig. 5.

Fig. 4. Dependence of the maximum deviation angle

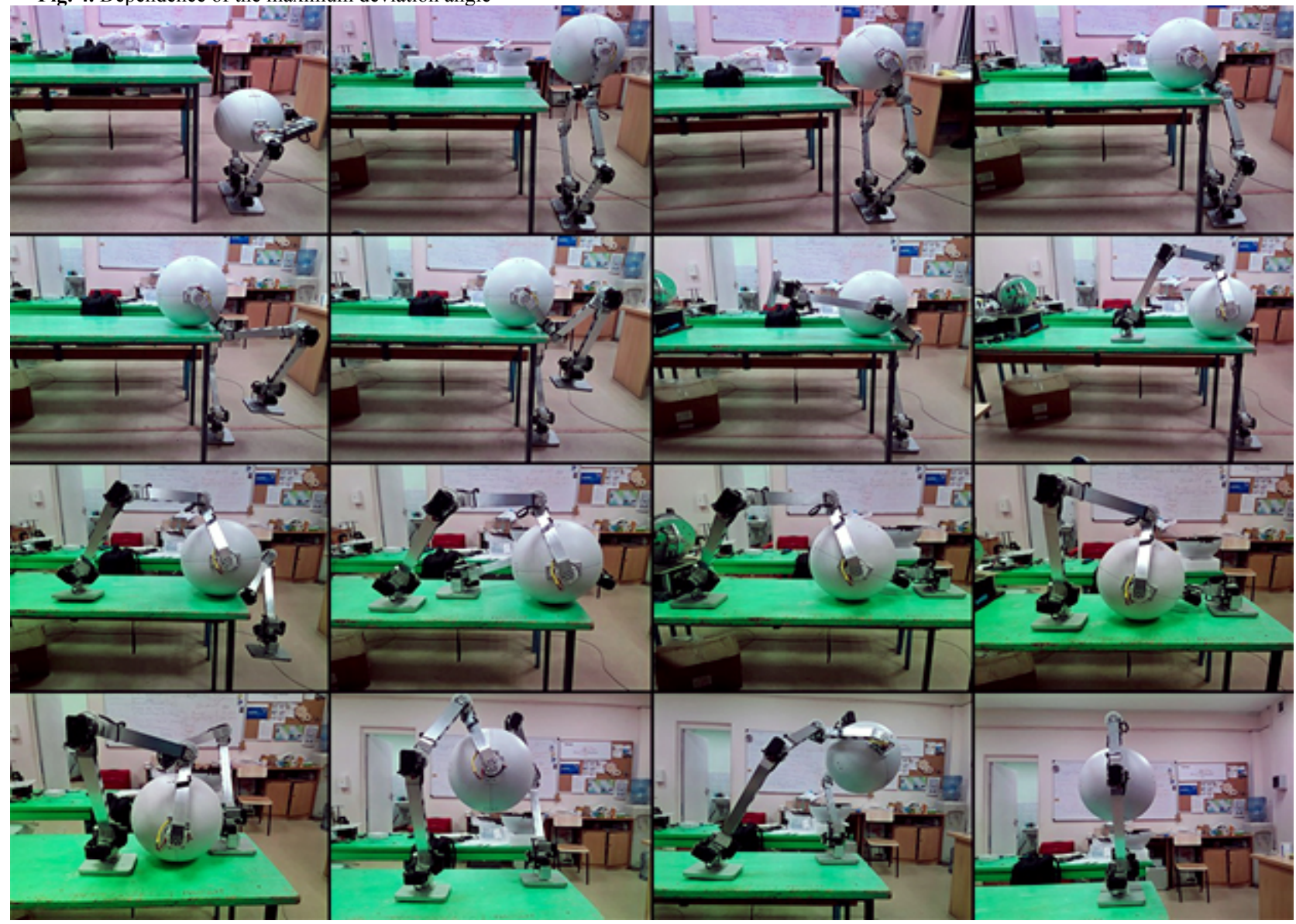

Fig. 5. Phases of overcoming the robot step, with a height equal to the height of the robot

have to work and move in complex terrain in- and outdoor, climb stairs, go to narrow spaces are the use of battery power and the ability to self-stabilize in various stochastic environment. The standard function of measuring robots'

\section{Conclusion}

The two criterions of effectiveness of walking robots which and other moving vehicles' efficiency in terms of moving is 
Cost of Transport (CoT), which is the non-dimensionalized energy criterion per unit weight and unit distance [13]. One of the main tasks of robotic engineers is the making the CoT parameter as low as possible. For example, the MIT Cheetah has a cost of transport around 0.5 , better than similar robots, and extremely close to its biological sources of inspiration [14]. On the one side of the problem there is the passive walking approach which can be highly efficient on determined, often flat surface, but very hulking and unstable within unpredictive conditions, i.e. the Cornell biped which seems to be the most efficient legged machine built today [15]. But such robots usually are scientific experiments and are unable to solve any kind of utility task in real world. Vast majority of walking robots use the same actuators for kinematics and stabilization just like humans and animals do. Some researchers try to optimize the design of electric motors and gears for the specific robots, the common approach is to use direct-drive actuators with elastic behavior [16].

For this robot, an experimental determination of the Cost of Transport (CoT) parameter was made [17]. It is also called specific tractive force or specific resistance (von Kármán-Gabrielli diagram), or the energy index [18]. In this case, the formula for the determination of CoT looks like:

$\cot =\frac{P}{m g V^{\prime}}$

where $\mathrm{P}$ is system power $(\mathrm{W}), \mathrm{V}$ is constant speed $(\mathrm{m} / \mathrm{s} 2)$.

AnyWalker specifications: average power consumption: $\mathrm{P}=110 \mathrm{~W}$; mass of the robot: $\mathrm{m}=16 \mathrm{~kg}$; travel speed on a horizontal surface: $\mathrm{V}=0.7 \mathrm{~m} / \mathrm{s}$.

Based on these data, the cost of moving AnyWalker on a flat horizontal surface is 1.0021 .

Depending on the speed, external conditions and other factors, the CoT can change when moving. These changes are related to the energy costs of the elements of the mobile platform. As a dimensionless value, CoT has a wide range of applications, allowing you to compare human, animal, transport, etc. Comparison of the AnyWalker platform with other known robots and moving objects is shown in Fig. 6.

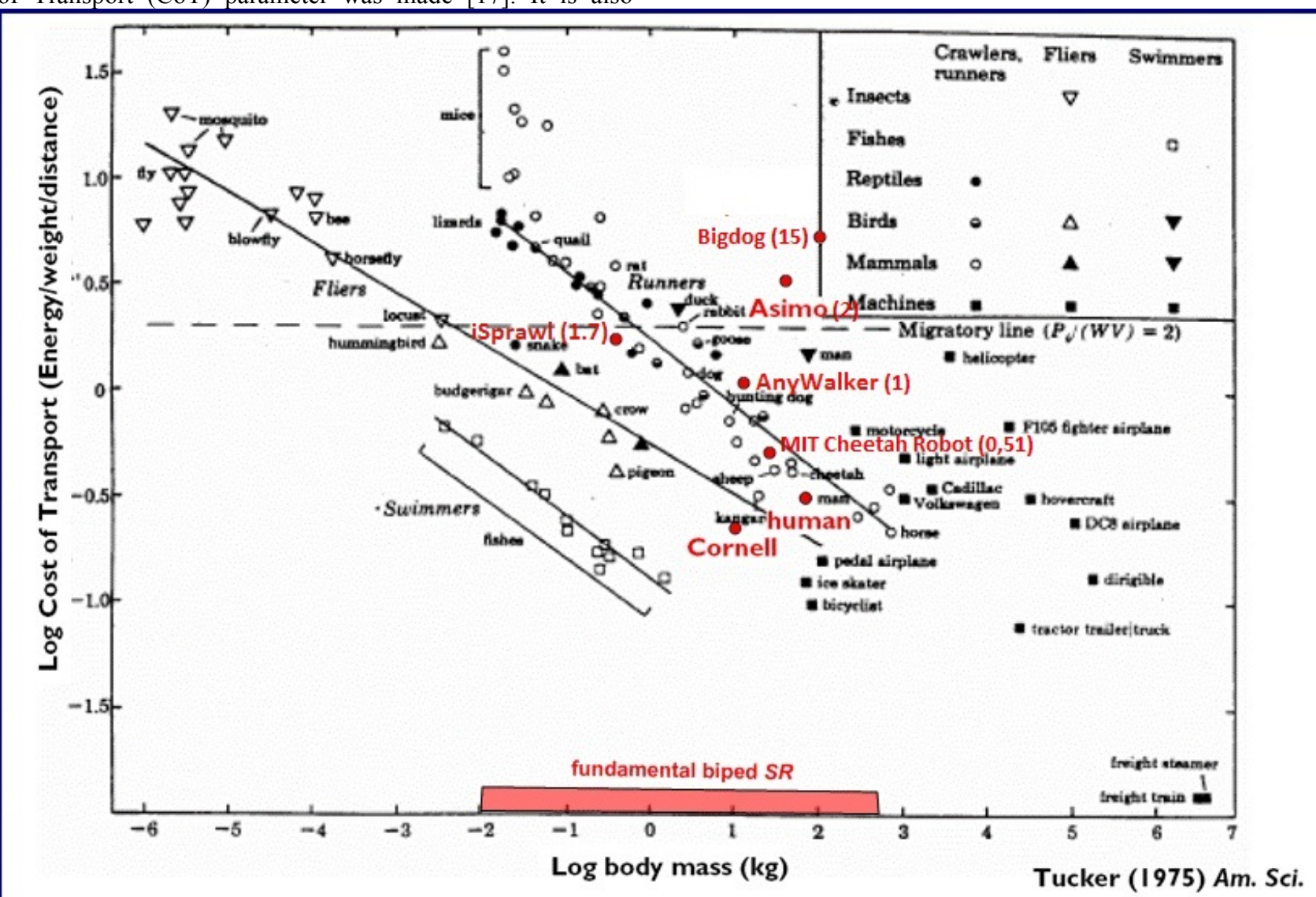

Fig. 6. Graph of the logarithm of the object mass from its energy cost of transportation.

Fig. 6 was first proposed in V. A. Tucker, 1975 to illustrate the comparison of the Cost of Transport parameters [17]. On the graph in the logarithmic scale there are values of the parameter CoT for living beings grouped by body weight (also in the logarithmic scale). Subsequently, this Fig. began to impose points for various robotic walking and driving vehicles $[14,19]$. Also for a comparative illustration of the energy efficiency of motion, the graphs of the reduced CoT are plotted against the mass and grouped according to the metabolism [20], as well as the CoT, depending on the running speed [21-23].

In this paper, we have shown that auxiliary stabilization system embedded in the robot's body expands the stability zone of a walking robot. It is useful during the transition from one motion phase to another, especially when the robot is supported by one leg only and in the case of backlash in the joints. Therefore, the introduction of the auxiliary internal stabilization system for biped robots is fully justified: it extends mobility, improves controllability and simplifies the mathematical modeling of dynamic walking.

\section{Acknowledgements}

This work was supported by state task of the Russian Ministry of Education and Science, project No. 8.2321.2017 
"Development and adaptation of control systems for compensation of dynamic deflecting effects on mobile objects in a state of dynamic equilibrium".
This is an Open Access article distributed under the terms of the Creative Commons Attribution License

\section{References}

1. D.C. Kar, Design of a statically stable walking robot: A review, Journal of Robotic Systems, Vol. 20 (Issue 11):671-686, 2003.

2. A. Goswami, Walking Robots. Encyclopedia of Systems and Control, pp. 1537-1548, 2015.

3. T. Booysen, S. Marais The development of a remote controlled, omnidirectional six legged walker with feedback. AFRICON 2013, pp. 1-6, 2015.

4. F. Tedeschi, G. Carbone, Design issues for hexapod walking robots. Robotics, Vol. 3(Issue 2):181-206, 2014.

5. H. Christensen, A Roadmap for US Robotics from Internet to Robotics, 2016 edn. (National Science Foundation \& University of California, San Diego, 2016)

6. X. Xiong, F. Wörgötter, P. Manoonpong, Adaptive and energy efficient walking in a hexapod robot under neuromechanical control and sensorimotor learning. IEEE transactions on cybernetics, Vol. 46(Issue 11):2521-2534, 2016.

7. N.S. Szczecinski, A.J. Hunt, R.D. Quinn, Design process and tools for dynamic neuromechanical models and robot controllers. Biological cybernetics, Vol. 111(Issue 1): 105-127, 2017

8. J. Zhao, Q. Liu, S. Schütz, K. Berns, Experimental verification of an approach for disturbance estimation and compensation on a simulated biped during perturbed stance. In 2014 IEEE International Conference on Robotics and Automation (ICRA), pp. 5082-5087, 2014.

9. G.H. Liu, H.Y. Lin, H.Y. Lin, S.T. Chen, P.C. Lin, Design of a kangaroo robot with dynamic jogging locomotion. In 2013 IEEE/SICE International Symposium on System Integration (SII), pp. 306-311, 2013.

10. J. Borràs, \&, A. M. Dollar, Static analysis of parallel robots with compliant joints for in-hand manipulation. In 2012 IEEE/RSJ International Conference on Intelligent Robots and Systems (IROS), pp. 3086-3092, 2012.

11. I. Ryadchikov, , E. Nikulchev, S. Sechenev et al. Development of robotic mobile platform with the universal chassis system. IOP Conference Series: Materials Science and Engineering, Vol. 312, p. $012021,2018$.

12. M. Gajamohan, M. Muehlebach, Widmer, R. D'Andrea. The Cubli: A reaction wheel based 3D inverted pendulum. 2013 European Control Conference (ECC), pp. 268-274, 2013.
13. T. Yang, Control of aperiodic walking and the energetic effects of parallel joint compliance of planar bipedal robots, Ph.D. dissertation, The Ohio State University, 2007.

14. S. Seok, A. Wang, M.Y.M. Chuah et al. Design principles for energy-efficient legged locomotion and implementation on the MIT Cheetah robot. IEEE/ASME Transactions on Mechatronics, Vol. 20(Issue 3), pp. 1117-1129, 2015.

15. D. Zarrouk, R.S. Fearing. Cost of locomotion of a dynamic hexapedal robot. In 2013 IEEE International Conference on Robotics and Automation (ICRA), pp. 2548-2553, 2013.

16. F. Bauer, U. Römer, A. Fidlin, W. Seemann. Optimization of energy efficiency of walking bipedal robots by use of elastic couplings in the form of mechanical springs. Nonlinear Dynamics, Vol. 83(Issue 3):1275-1301, 2016.

17. V.A. Tucker. The energetic cost of moving about: walking and running are extremely inefficient forms of locomotion. Much greater efficiency is achieved by birds, fish - and bicyclists. American Scientist, Vol. 63(Issue 4):413-419, 1975.

18. W. Shi, D. Stapersma, H.T. Grimmelius. Comparison study on moving and transportation performance of transportation modes. International Journal of Energy and Environment, Vol. 2(Issue 4): 179-190, 2008.

19. J.C. Larsen, K. Stoy. Energy efficiency of robot locomotion increases proportional to weight. Procedia Computer Science, Vol. 7:228-230, 2011.

20. R.M. Alexander. Models and the scaling of energy costs for locomotion. Journal of Experimental Biology, Vol. 208(Issue 9): 1645-1652, 2005.

21. M. Khoramshahi, A. Spröwitz, A. Tuleu et al. Benefits of an active spine supported bounding locomotion with a small compliant quadruped robot. In 2013 IEEE International Conference on Robotics and Automation (ICRA), pp. 3329-3334, 2013.

22. A.D. Kuo, J.M. Donelan, A. Ruina. Energetic consequences of walking like an inverted pendulum: step-to-step transitions. Exercise and sport sciences reviews, Vol. 33(Issue 3): 88-97, 2005.

23. J.C. Selinger, S.M. O'Connor, J.D. Wong, J.M. Donelan. Humans can continuously optimize energetic cost during walking. Current Biology, Vol. 25(Issue 18):2452-2456, 2015. 